\title{
Implementation of 2D Active Shape Model-based Segmentation on Hippocampus
}

\author{
Yonny S. Izmantoko ${ }^{\dagger}, \mathrm{Ho}^{-S u n g}$ Yoon $^{+\dagger}$, Enkhbolor Adiya ${ }^{++\dagger}$, \\ Chi-Woong Mun ${ }^{+++}$, Young Huh ${ }^{++++}$, Heung-Kook Choi ${ }^{++++++}$
}

\begin{abstract}
Hippocampus is an important part of brain which is related with early memory storage and spatial navigation. By observing the anatomy of hippocampus, some brain diseases effecting human memory (e.g. Alzheimer, schizophrenia, etc.) can be diagnosed and predicted earlier. The diagnosis process is highly related with hippocampus segmentation. In this paper, hippocampus segmentation using Active Shape Model, which not only works based on image intensity, but also by using prior knowledge of hippocampus shape and intensity from the training images, is proposed. The results show that ASM is applicable in segmenting hippocampus from whole brain MR image. It also shows that adding more images in the training set results in better accuracy of hippocampus segmentation.
\end{abstract}

Key words: Active shape model, hippocampus, segmentation, MRI

※ Corresponding Author: Heung-Kook Choi, Address : (621-749) Department of Computer Engineering, Inje University Injero-197, Gim-Hae Gyeong-Nam Rep. of Korea, TEL : +82-055-320-3437, FAX : +82-055-3223107, E-mail : cschk@inje.ac.kr

Receipt date: Oct. 1, 2013, Approval date : Nov. 12, 2013

${ }^{+}$Dept. of Computer Engineering, UHRC, Inje University, Korea

(E-mail: yonny.septian@gmail.com)

${ }^{++}$Dept. of Computer Engineering, UHRC, Inje University, Korea

(E-mail: evehoti@naver.com)

${ }^{+++}$Dept. of Computer Engineering, UHRC, Inje University, Korea

(E-mail: enkhbolora@yahoo.com)

${ }^{++++}$Dept. of Biomedical Engineering, Inje University, Korea

(E-mail:mcw@inje.ac.kr)

${ }^{++++}$Korea Evaluation Institute of Industrial Technology, Korea (E-mail: yhuh@keit.re.kr)

${ }^{++++++}$Dept. of Computer Engineering, UHRC, Inje University, Korea

※ This research was supported by Basic Science Research Program through the National Research Foundation of Korea (NRF) funded by the Ministry of Education, Science, and Technology (2012-0002646). Also thanks to Haeundae Paik Hospital, Dong-A University Hospital, Pusan National University Hospital, and Gyeongsang National University Hospital for providing the MR images for this research.

\section{INTRODUCTION}

Dementia is a brain disease which characterized by the loss of global cognitive ability. Usually it happens because of aging, but there is also a possibility that it happens before the age of 65 , which is called early onset dementia. Dementia is the sec $^{-}$ ond leading health concern among adults (after cancer) [1]. The common cause of dementia in the elderly is Alzheimer Disease ( $\mathrm{AD}$ ). AD accounts for more than $40 \%$ of dementia in the elderly [2]. Early $\mathrm{AD}$ can be detected using human brain volumetric techniques. In order to detect early $\mathrm{AD}$, the anatomy of hippocampus, which is the part of the brain that is involved in memory forming, organiz ${ }^{-}$ ing, and storing, is needed to be obtained from whole brain image.

In MR image, the intensity of hippocampus is difficult to be distinguished with the intensity value of other tissues in its surrounding. Therefore, segmentation method that only works based on intensity value cannot be used directly. Several methods in previous researches have been applied to segment hippocampus from the whole brain MR 
image. Jang, D.P. et al combined dynamic contour model and region growing [3], Xiang Lu et al used watersnake [4], which is a combination of watershed and snake algorithm. D. Shen et al proposed hippocampus segmentation using a deformable shape model [5], and M. Chupin et al used probabilistic atlas to segment hippocampus automatically [6]. In this paper, another approach of hippocampus segmentation called active shape model is performed. A set of images were used for training and an image was used for testing the method.

\section{ASM IMPLEMENTATION}

Active shape model (ASM) is a method proposed by T.F.Cootes et al in 1994 [7]. This method uses prior knowledge from several sample images. This knowledge is useful to deal with object which varies in size and shape. In ASM, we use data from each sample image to make a model which will be used to segment another image [8]. There are four main steps of this method: training, modeling, initialization, and segmentation.

\subsection{Training}

The goal of this step is to make a training set consisting of hippocampus shapes which are $\mathrm{ex}^{-}$ pressed by the coordinate of markers. This is the most time-consuming process of ASM because of the manual placement of markers. In our hippocampus case, we put 11 markers on the hippocampus. The order of marker is shown in Fig. 1. The markers were put on the most important and

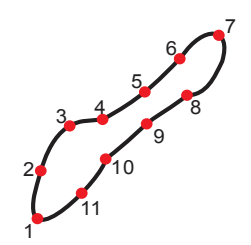

Fig. 1. The order of markers placement on hippocampus. distinguishable parts of object, e.g. corner, curve, etc.

Even though 11 markers have been put on the most important parts of object, they still cannot represent the actual shape of hippocampus. Therefore, we put more markers between existing markers automatically using interpolation. The goal of this interpolation process was not only to get better shape of hippocampus, but also to observe the effect of marker numbers in the ASM segmentation.

The shape of hippocampus was represented by a set of $\mathrm{n}$ points. These points were represented by a vector $\vec{x}_{i}$ in(1), where $\vec{x}_{i}$ is the number of markers and $i$ is the number of sample images

$$
\vec{x}_{i}=\left(x_{i 1}, y_{i 1} ; x_{i 2}, y_{i 2} ; \ldots ; x_{i n}, y_{i n}\right)^{T}
$$

\subsection{Modeling}

ASM works by examining the statistics of the coordinates of the labeled points over the training set. The variation of hippocampus position on each sample images results in different location of the markers coordinate. Therefore, it is necessary to align all shapes acquired from training step before making any statistical computation. There are several methods of shape alignment; one of them is Generalized Procrustes Analysis (GPA) which is used to align a set of object [9]. In our work, we moved every shape to the base of the image coordinate before applying any transformation. It was done by updating the value of each coordinate in a shape by the mean value of all coordinates in that shape (translation offset).

Variation of object orientation can be removed using the direction of each point in a shape. We compute the mean direction of the shape (rotation offset) and uses it to update the direction of each point. The last step of this alignment is to make new points with updated directions that will be used for further statistical computation.

After aligning all shapes in the training set, we 


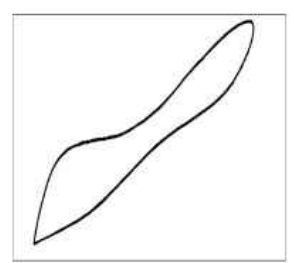

Fig. 2.The mean shape model of hippocampus.

computed the mean shape of the training set by (2), where $x_{i}$ is the vector of all coordinate in a shape and $\mathrm{n}$ is the number of shapes used in the training set.

$$
\bar{x}=\frac{1}{n} \sum_{i=1}^{n} x_{i}
$$

Fig. 2 shows the mean shape of hippocampus acquired from the training set. This shape was the base of all shape variation of ASM. The variations were made by the movement of each markers based on eigenvalue and eigenvector.

Covariance matrix of all training data was computed by (3). From this covariance matrix, we obtained the eigenvalue $\lambda_{i}$ and eigenvector $\vec{v}_{i}$. It is always possible to get too many eigenvalues from the computation. Too many eigenvalues means too many variations that can be made by the mean shape, and it can result in confusion in segmentation process. To limit the number of eigenvalues, we only use some significant eigenvalues which values are bigger than $98 \%$ of total eigenvalues.

$$
S=\frac{1}{n} \sum_{i=1}^{n}\left(\vec{x}_{i}-\bar{x}\right)\left(\vec{x}_{i}-\bar{x}\right)^{T}
$$

The model can be approximated by using mean shape, significant eigenvectors, and weight vector ${ }_{\vec{w}}(4)$, where $\vec{V}=\left(\vec{v}_{1}, \vec{v}_{2}, \ldots, \vec{v}_{i}\right)$. The value of $\vec{w}_{i}$ is bounded by the value of eigenvalue $\lambda_{i}$ by (5) The variations of mean shape by some eigenvalues are shown in Fig. 3. The first parameter adjusts the lower curvature direction of hippocampus. The second and the fourth parameters vary the length of the main shape. The third parameter changes the thickness of the lower part of hippocampus. The fifth and sixth parameters vary the shape of

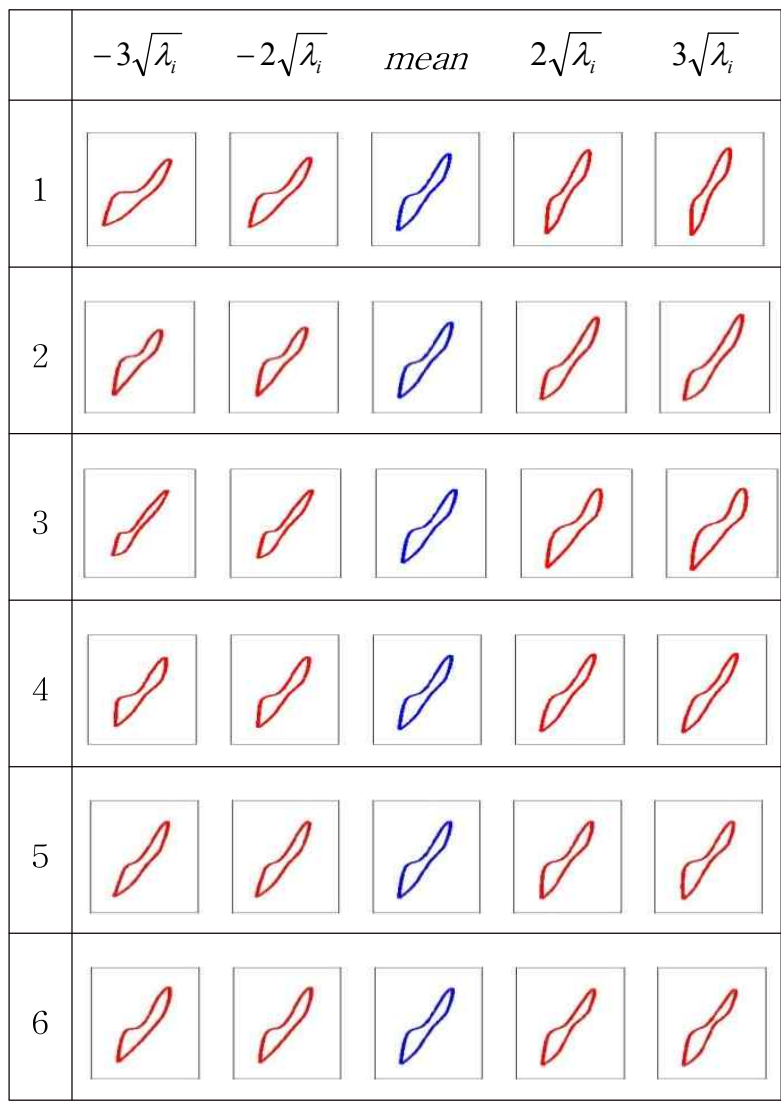

Fig. 3. The mean shape variations of hippocampus.

upper and lower part of mean shape, respectively.

$$
\begin{aligned}
& x=\bar{x}+\vec{V} \vec{w} \\
& -3 \sqrt{\lambda_{i}} \leq w_{i} \leq 3 \sqrt{\lambda_{i}}
\end{aligned}
$$

The next step is to make gray level appearance. Its function is to get the mean gray level profile along the normal of each marker point. We choose $\mathrm{k}$ points outside the marker point and $\mathrm{k}$ points inside the marker point. Therefore, we have $2 \mathrm{k}^{+1}$ gray level profile for each marker. For all shapes in the training set, we will get the mean gray profile.

\subsection{Initialization}

This is the process to place the mean shape to the image that we want to segment. It is necessary to place the mean shape as precise as possible to the object that we want to segment [10] Misplacing the mean shape usually results in 
wrong segmentation.

\subsection{Segmentation}

Segmentation is the last step in ASM. The mean shape evolves by the movement of each marker. The distance of point movement in the normal is defined as the gray level resolution [11]. This resolution can be vary from coarse (far distance between each profile points) to fine (short distance between each profile points). This each point moves along its normal by minimizing the Mahalanobis distance between the mean gray profile and the gray profile of the image (6) [12]. The segmentation is expected to converge. However, specific iteration number was used in the segmentation process to ensure the stop condition of this method.

$$
f\left(g_{i}\right)=\left(g_{i}-\bar{g}\right) S_{g}^{-1}\left(g_{i}-\bar{g}\right)
$$

\section{EXPERIMENTAL RESULTS}

The input and training images were DICOM files obtained from $1.5 \mathrm{~T}-3 \mathrm{~T}$ MRI devices from four university hospitals in Korea. $12 \mathrm{MR}$ brain sets obtained from 3 volunteer students from 4 university hospitals. For each image set, 2 to 3 slices which correctly resembles the shape of hippocampus were used to make the model.

The ASM segmentation result is influenced by some parameters: the number of images in the training set, the number of markers, the initialization coordinate, and the number of pixels along the normal of each marker as gray level profile. In our work, the initialization position and the number of gray level profile were fixed. The modified variables were the number of training images and the number of markers. The segmentation result superimposed on the image is shown in Fig. 4a and the hippocampus is shown in Fig. $4 \mathrm{~b}$. In order to get the accuracy of hippocampus segmentation using ASM, we use the difference between the ASM segmentation result and manual segmentation re-

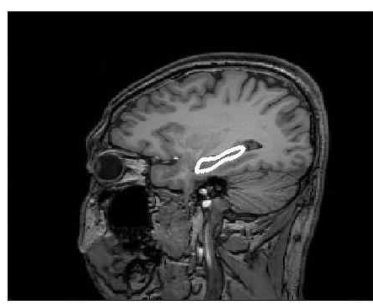

(a)

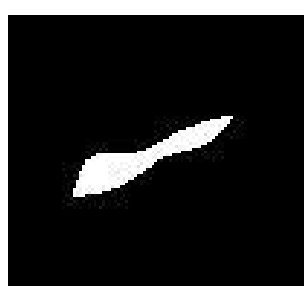

(b)

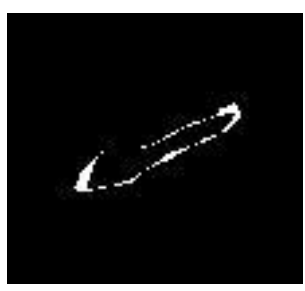

(c)
Fig. 4. (a) the segmentation of hippocampus; (b) the area of hippocampus; (c) the difference between ASM and manual segmentation.

sult (Fig. 4c). The manual segmentation was done by doing delineation along the boundary of hippocampus. The accuracy is presented as error percentage. Table 1 and Table 2 show the effect of the number of markers and the number of sample image, respectively.

We used point to point interpolation to get more marker points. 5 types of interpolation; by 5,10 , 15, 20, and 25 points were used, while preserving other parameters (number of image samples,

Table 1. Influence of the number of interpolated points between markers

\begin{tabular}{|c|c|c|c|}
\hline $\begin{array}{c}\text { Interpolated } \\
\text { points }\end{array}$ & $\begin{array}{c}\text { Total marker } \\
\text { points }\end{array}$ & $\begin{array}{c}\text { Time elapsed } \\
(\mathrm{s})\end{array}$ & $\begin{array}{c}\text { Error } \\
(\%)\end{array}$ \\
\hline \hline 5 & 29 & 4.79 & 30.272 \\
\hline 10 & 64 & 5.78 & 25.170 \\
\hline 15 & 99 & 6.87 & 28.912 \\
\hline 20 & 134 & 7.75 & 26.701 \\
\hline 25 & 169 & 8.66 & 24.830 \\
\hline
\end{tabular}

Table 2. Influence of the number of image samples in training set

\begin{tabular}{|c|c|c|}
\hline $\begin{array}{c}\text { Number of sample } \\
\text { images }\end{array}$ & Time elapsed & Error (\%) \\
\hline \hline 10 & 8.75 & 49.523 \\
\hline 20 & 8.73 & 35.612 \\
\hline 30 & 8.71 & 27.139 \\
\hline 40 & 8.66 & 24.830 \\
\hline
\end{tabular}


length of gray profile, and initialization coordinate) Our hippocampus model with more points needs more time to finish the segmentation process. However, the accuracy of model with more points is not always better than model with fewer points. In our result, interpolation by 10 points was the optimum result.

The number of sample images in the training set will also have a direct influence to the segmentation result. More sample images mean richer variations of the model, thus, make the model more applicable in the target image. Different number of sample images were used; 10, 20, 30 and 40. The number of interpolated points was set to 25 for all segmentation process and the initialization coordinate was fixed. This result shows that more sample images results in better segmentation $\mathrm{re}^{-}$ sult, without any significant difference in time needed for segmentation.

\section{CONCLUSION}

Active shape model is applicable to segment hippocampus from whole brain image. The best accuracy was achieved by using 40 images in the training set. This result can be improved by employing medical experts in placing the markers on the training images to make the model and doing the manual segmentation.

In addition, to increase the segmentation accuracy, it is encouraged to add more sample images in the training set instead of adding more markers on the object. If the object model already represents the hippocampus shape, we do not need to add more markers since it will only add the computation time. On the other hand, adding more sample images results in richer variations of the model and better segmentation accuracy.

For further work, along with the medical experts, not only two-dimensional ASM, but also three-dimensional (3D) ASM can be performed. By using more images in the training set, more precise placement of markers in the training set, and correct manual segmentation, a good accuracy for $3 \mathrm{D}$ hippocampus segmentation can be achieved.

\section{REFERENCES}

[1] International Survey Highlights Great Public Desire to Seek Early Diagnosis of Alzheimer's, http://www.hsph.harvard.edu/news/pressreleases/alzheimers-international-survey, 2013.

[2] Y.Y. Sitoh, J. Karthik, J.C. Rajapakse, W.T. Hong, W.L. Lee, S. Sahadevan, and J.J. Chin, "Cerebral and Hippocampal Volumetry in Early Alzheimer'sdisease," Proc of the $g^{\text {th }}$ Int. Conf. on Neural Inf. Process., Vol. 3, pp. 15231526, 2002.

[ 3 ] D.P. Jang, D.S. Lee, and S.I. Kim, "Contour detection of Hippocampus using Dynamic contour Model and Region Growing," Proc. of the $19^{\text {th }}$ Annu. Int. Conf of the IEEE on Eng. in Med. and Biol. Soc., Vol. 2, pp. 763-766, 1997.

[4] X. Lu and S. Luo, "Segmentation of Hippocampus in MRI using Watersnakes," IEEE Int. Conf. on Complex Med. Eng., pp. 552-555, 2007.

[5] D. Shen, S. Moffat, S. M. Resnick, and C. Davatzikos, "Measuring Size and Shape of the Hippocampus in MR Imagesusing a Deformable Shape Model," NeuroImage, Vol. 15, No. 2, pp. 422-434, 2002.

[6] M. Chupin, G. Chetelat, L. Lemieux, B. Dubois, L. Garnero, H. Benali, F. Eustache, S. Lehericy, B. Desgranges, and O. Colliot, "Fully Automatic Hippocampus Segmentation Discriminates between Early Alzheimer's Disease and Normal Aging," $5^{\text {th }}$ IEEE Int. Symp. on Biomed. Imaging, pp. 97-100, 2008.

[ 7 ] T.F. Cootes, C.J. Taylor, D.H. Cooper, and J. Graham, "Active Shape Models - Their Training and Application," Comput. Vis. and 
Image Underst., Vol. 51, No. 1, pp. 38-59, 1995.

[8] A. Enkhbolor; I. Yonny S.; H.K. Choi, H, "Comparison of Active Contour and Active Shape Approaches for Corpus Callosum Segmentation," J. Korea Multimedia Soc, Vol. 16, No. 9, pp. 1018-1030, 2013.

[9] T.F. Cootes and C.J. Taylor, Statistical Models of Appearance for Computer Vision, University of Manchester, Manchester, 2004.

[10] S. Nyamlkhagva, S. Altansukh, A. Enkhbolor, H.K. Choi, "Contrast Enhancement for Segmentation of Hippocampus on Brain MR
Images," J. Korea Multimedia Soc, Vol. 15, No. 12, pp. 1409-1416, 2012.

[11] B. van Ginneken, A.F. Frangi, J.J. Staal, B.M. ter Haar Romeny, and M.A. Viergever, "Active Shape Model Segmentation with Optimal Features," IEEE Trans. on Med. Imaging, Vol. 21, No. 8, pp. 924-933, 2002.

[12] M. Benjelloun, S. Mahmoudi, and F. Lecron, "A Framework of Vertebra Segmentation using the Active Shape Model-Based Approach," Int. J. of Biomed. Imaging, Vol. 2011, No. 9, pp. 1-14, 2011. 


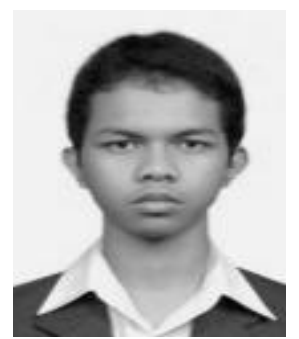

\section{Yonny Septian Izmantoko}

received the B.S. degree in Electrical Engineering from Institut Teknologi Bandung, Indonesia, in 2011. He was theformer member of Medical Image Technology Laboratory (MITL), Inje University, who has received his master of Computer Science degree in 2013. His research interests are image segmentation, image visualization, and parallel data computing using GPU.

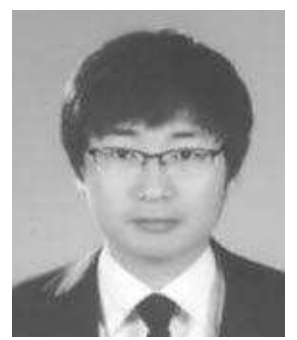

\section{Ho-SungYoon}

received the B.S. degree in $\mathrm{Com}^{-}$ puter Engineering from Inje University in 2012. He is currently a master student in $\mathrm{Med}^{-}$ ical Image Technology Laboratory (MITL), School of $\mathrm{Com}^{-}$ puter Engineering, Inje University. His research interests are image analysis and image visualization.

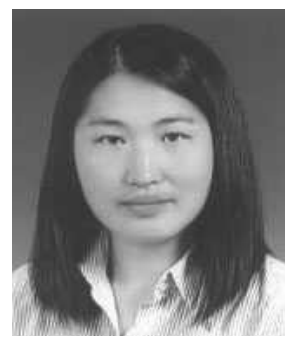

\section{Enkhbolor Adiya}

received the B.S., M.S. degrees in Applied Mathematics from School of Mathematics and Computer Science of National University of Mongolia in 2004, 2006 respectively. She is currently a $\mathrm{PhD}$ student in Medical Image Technology Laboratory (MITL), School of Computer Engineering, Inje University, South Korea. Her research interests include image restoration and image segmentation.

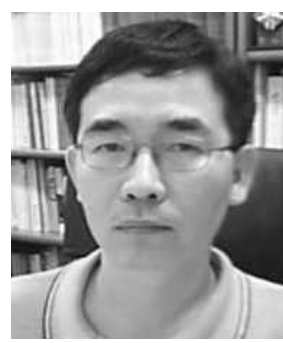

\section{Chi-Woong Mun}

received the M.S. and Ph.D. degrees in Electrical Engineering from Korea Institue of Science and Technology, Korea in 1983 and 1991, respectively. He had been a senior researcher of Asan Institute of Life Science (19911995) and principal researcher of Samsung Advanced Institute of Technology (1996-1998). Now he is an associate professor in department of Biomedical Engineering, Inje University. His interesting research fields are in signal and medical imaging system, biomedical instruments, and so on.

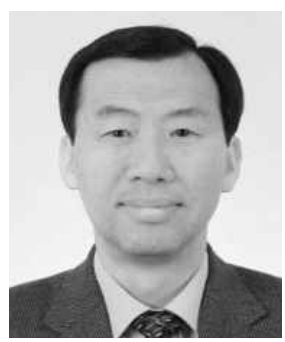

\section{Young Huh}

received $\mathrm{Ph} . \mathrm{D}$. degree from Department of Electrical Engineering, The University of Texasin 1995 andwas Visiting Scholar at University of Washington Medical Center in 2005. He was Director of Medical IT Fusion Research Division at Korea Electrotechnology Research Institute (KERI) andhe is currently working as Medical Devices Program Director at Korea Evaluation Institute of Industrial Technology(KEIT) and Chairman of Korea IEC/TC 62 Standard Technical Expert Committee (Korean Agency for Technology and Standards). His interesting research fields are medical imaging devices and system, and semiconductor sensors.

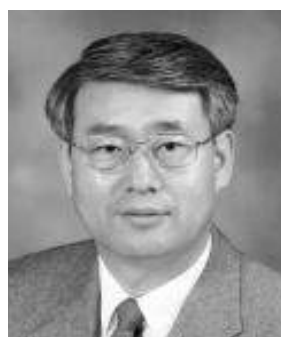

\section{Heung-Kook Choi}

has gone the undergraduate studying and graduate studying in computer science and engineering at the Department of Electrical Engineering of Linköping University, Sweden (1984-1990) and Ph.D. studying in computerized image analysis at the Center for Image Analysis of Uppsala University, Sweden (1990-1996). He was President of Industry and Academic Cooperation Foundation atInje University and now he is President of Korea Multimedia Society. His interesting research fields are in computer graphics, virtual reality, and medical image processing and analysis. 